\section{Massive Gastrointestinal Hemorrhage in a Renal Transplant Recipient Due to Visceral Kaposi's Sarcoma}

\begin{abstract}
A 15-year-old girl who had received a cadaver renal allograft, was discharged from the transplantation unit 12 days after the operation, with immunosuppressive therapy. The patient was readmitted 3 months later, due to the appearance of diarrhea and fever associated with lymphadenopathy, hepatosplenomegaly and severe recurrent episodes of lower gastrointestinal bleeding.
\end{abstract}

An emergency colonoscopy revealed the presence of multiple nonbleeding maculopapular lesions with a central umbilication along the entire colonic mucosa (Figure 1). An upper gastrointestinal endoscopy disclosed fresh blood and clots in the stomach and the presence of numerous oozing large polypoid volcano-like lesions (Figure 2) within the corpus and the fundus. The endoscopic examination was completed by the performance of multiple biopsies at the edges of the lesions, which showed findings consistent with Kaposi's sarcoma.

After the diagnosis of visceral Kaposi's sarcoma, withdrawal of immunosuppressive therapy for 20 days completely stopped all bleeding after 16 days. An endoscopic inspection performed 1 month after the last bleeding, showed a decrease in the size and number of the lesions, with complete remission 6 months later.

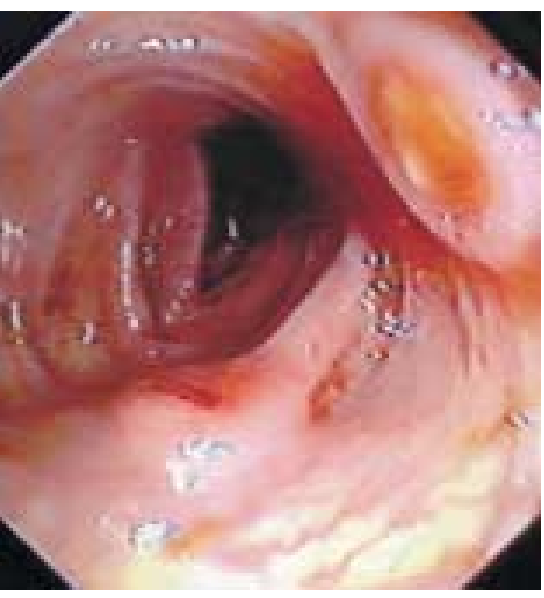

Figure 1 Multiple maculopapular lesions observed along the colonic mucosa
Kaposi's sarcoma and lymphoproliferative disorders are the most commonly reported malignancies occurring in transplant recipients; in this population, $6 \%$ of all observed cancers are Kaposi's sarcoma [1]. Massive gastrointestinal bleeding is the most common clinical manifestation of gastrointestinal involvement of Kaposi's sarcoma and is considered a sign of small bowel involvement [2]. We cannot exclude the possibility that in our patient the hemorrhage began from the ileum, even though we found actively bleeding lesions in the stomach.

Treatment of Kaposi's sarcoma in renal transplant recipients consists of the reduction or cessation of immunosuppressive therapy [3]. In two recent series [4, 5] the withdrawal of such therapy allowed a complete remission of the disease in $61 \%$ of patients, as in our patient, 69 and $68 \%$ of the patients in the series remained dialysis-free.

\section{A. Calzona ${ }^{1}$, P. Naso $^{1}$, C. Puliatti ${ }^{2}$,}

P. F. Veroux ${ }^{2}$, F. Leone $^{2}$, A. Russo ${ }^{1}$

${ }^{1}$ Endoscopy Unit, Dept. of Surgery,

Policlinico Universitario Catania, Italy

${ }^{2}$ Kidney Transplant Unit, Dept. of Surgery,

Policlinico Universitario Catania, Italy

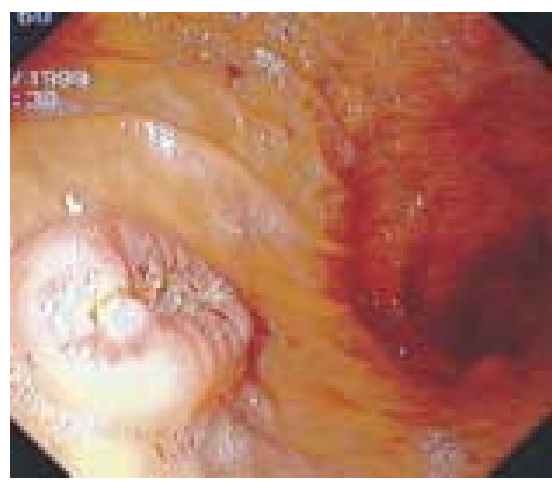

Figure 2 A polypoid volcano-like lesion located on the corpus of the stomach

\section{References}

${ }^{1}$ Penn I. Incidence and treatment of neoplasia after transplantation. J Heart Lung Transplant 1993; 12: 328 - 336

${ }^{2}$ Ablin J, Acherman Z, Eliakim R. Diffuse gastrointestinal hemorrhage as a presentation of systemic Kaposi sarcoma. Am J Gastroenterol 1998; 93: $1390-$ 1391

${ }^{3}$ Shepherd FA, Maher E, Cardella C, et al. Treatment of Kaposi's sarcoma after solid organ transplantation. J Clin Oncol 1997; 15: 2371 - 2377

${ }^{4}$ Qunibi W, Akhtar M, Sheth K, et al. Kaposi sarcoma: the most common tumor after renal transplantation in Saudi Arabia. Am J Med 1988; 84: 225-232

${ }^{5}$ Montagnino G, Bencini PL, Tarantino A, et al. Clinical features and course of Kaposi's sarcoma in kidney transplant patients: report of 13 cases. Am J Nephrol 1994; 14: $121-126$

\section{Corresponding Author}

A. Russo, M.D.
Policlinico-Università di Catania
Servizio di Chirurgia Endoscopica
Via S. Sofia, 84
95100 Catania
Italy
Fax: +39-095-337065
E-mail: arusso@mbox.unict.it

